\title{
FRACTAL APPROXIMATION OF JACKSON TYPE FOR PERIODIC PHENOMENA
}

\author{
M. A. NAVASCUÉS, ${ }^{*} \S$ SANGITA JHA, ${ }^{\dagger}$ A A. K. B. CHAND ${ }^{\dagger, \|}$ \\ and M. V. SEBASTIÁN ${ }^{\ddagger}, * *$ \\ *Departamento de Matemática Aplicada \\ Escuela de Ingeniería y Arquitectura \\ Universidad de Zaragoza, Zaragoza 500018, Spain \\ ${ }^{\dagger}$ Department of Mathematics \\ Indian Institute of Technology Madras \\ Chennai 600036, Tamil Nadu, India \\ ${ }^{\ddagger}$ Centro Universitario de la Defensa \\ Academia General Militar, Zaragoza 50090, Spain \\ \$manavas@unizar.es \\ 『sangitajha285@gmail.com \\ "lchand@iitm.ac.in \\ **msebasti@unizar.es
}

Received April 4, 2018

Accepted June 10, 2018

Published October 5, 2018

\begin{abstract}
The reconstruction of an unknown function providing a set of Lagrange data can be approached by means of fractal interpolation. The power of that methodology allows us to generalize any other interpolant, both smooth and nonsmooth, but the important fact is that this technique provides one of the few methods of nondifferentiable interpolation. In this way, it constitutes a functional model for chaotic processes. This paper studies a generalization of an approximation formula proposed by Dunham Jackson, where a wider range of values of an exponent of the basic trigonometric functions is considered. The trigonometric polynomials are then transformed in close fractal functions that, in general, are not smooth. For suitable election of this
\end{abstract}

\footnotetext{
${ }_{\text {Corresponding author. }}$
} 
M. A. Navascués et al.

parameter, one obtains better conditions of convergence than in the classical case: the hypothesis of continuity alone is enough to ensure the convergence when the sampling frequency is increased. Finally, bounds of discrete fractal Jackson operators and their classical counterparts are proposed.

Keywords: Fractals; Fractal Interpolation; Trigonometric Approximation; Convergence; Smoothing; Curve Fitting.

\section{INTRODUCTION}

Nearly all the scientists working in real-world applications must solve a problem of approximation at some point of their professional activity. A large number of publications consider this mathematical problem. One of the reasons of this phenomenon is the essential contribution of computers for the solution of numerical problems. Although the classical Fourier methods remain extremely useful tools, new techniques emerged in the last decades, such as wavelets and fractals (see for instance Ref. 1) are proven to be advantageous in the solutions of nonlinear phenomena in sciences and engineering.

Interpolation and approximation are mostly carried out with smooth functions, but in many practical situations we may come across nonsmooth experimental signals. Thus we cannot use any usual interpolation is such cases. Fractal interpolation helps to solve this problem to a large extent as it captures the nonlinearity feature associated with both smooth and nonsmooth models. The nonlinearity feature of these models or their derivatives depends on the choice of scaling parameters. Specific conditions on the scaling parameters for smoothness are given in Refs. 2-44. We give here a global deterministic method to model these signals by fractal interpolation. This method was introduced by Barnsley and co-worker. ${ }^{2[5] 6}$ For a given continuous function $f$ defined on a real compact interval, Navascués and Chand ${ }^{7}$ considered suitable iterated function system (IFS) to define a family of continuous function $f^{\alpha}$, which can interpolate and approximate $f$ properly. Several shape preserving properties of $f^{\alpha}$ and approximation properties of $\alpha$-fractal functions are studied in Refs. $8-10$.

This paper faces the generalization of a particular type of approximants defined by Jackson. 11 The second purpose is to define nonsmooth or smooth fractal versions of these classical approximants based on the choice of scale vector associated with them. This fact gives them a particular importance in order to obtain mathematical representations of experimental signals, from which only their values at evenly sampled nodes are known. For instance, time series recorded with a constant sampling frequency may be processed by means of these procedures. A possible application of the studied methods is the computation of integral parameters, for instance, the power of an experimental signal. However, if the number of points is large and/or they are noisy, the approximation is better than interpolation in many cases.

The uniform error bounds between the original function in $\mathcal{C}[-\pi, \pi]$ and its fractal Jackson approximants are obtained based on the range of a positive exponent parameter. The proposed limits prove the convergence of the fractal analog of the generalized discrete Jackson approximants with very weak conditions on the given function and using suitable choice of scale vector. Unlike the classical trigonometric case, the hypothesis of continuity alone is sufficient to provide the convergence of a generalized Jackson approximant when the number of terms tends to infinity. The fast evolution of the programs of advanced calculus enables the use of functions more complicated than mere polynomial and trigonometric mappings. In this way, from the theoretical point of view, new fractal nodal elements are proposed, that provide a generalization of the trigonometric functions. The density of these mappings in the space of continuous and periodic functions is proved. In some cases, the new fractal approximants perform better than the classical Jackson's originals. The paper is organized as follows. In Sec. 2, we give some brief description of $\alpha$-fractal functions and review the required classical results on uniform convergence of polynomial and trigonometric approximation. A generalization to nonsmooth fractal versions of discrete Jackson approximant is proposed and convergence results are studied for different ranges of exponent associated with the kernel function in Sec. 3. Finally, 
Sec. 4 is devoted to study the bound of discrete fractal Jackson operator.

\section{PRELIMINARIES}

In this section, we shall gather some essential materials on fractal function and trigonometric approximation. For a more detailed account, we refer the reader to Refs. 5,7 and 12,14 .

\subsection{Constructions of Fractal Functions}

The construction of continuous fractal interpolation function based on IFS is described by the following steps: Let $x_{1}<x_{2}<\cdots<x_{N}$ be real numbers, and $I=\left[x_{1}, x_{N}\right]$ be a closed interval that contains them. Let a set of data points $\left\{\left(x_{i}, y_{i}\right): i \in \mathbb{N}_{N}\right\}$ be given, where $\mathbb{N}_{k}$ is the first $k$ natural numbers. Let $L_{i}: I \rightarrow I_{i}:=\left[x_{i}, x_{i+1}\right], i \in \mathbb{N}_{N-1}$, be contractive homeomorphisms such that

$$
L_{i}\left(x_{1}\right)=x_{i}, \quad L_{i}\left(x_{N}\right)=x_{i+1} .
$$

Let $K=I \times \mathbb{R}$. Define $N-1$ continuous mappings $F_{i}: K \rightarrow \mathbb{R}$ such that

$$
\begin{aligned}
& F_{i}\left(x_{1}, y_{1}\right)=y_{i}, \quad F_{i}\left(x_{N}, y_{N}\right)=y_{i+1}, \\
& \left|F_{i}(x, y)-F_{i}\left(x, y^{\prime}\right)\right| \leq\left|\alpha_{i}\right|\left|y-y^{\prime}\right|
\end{aligned}
$$

where $(x, y),\left(x, y^{\prime}\right) \in K, \alpha_{i} \in(-1,1), i \in \mathbb{N}_{N-1}$. Now define functions $w_{i}: \mathbb{R}^{2} \rightarrow \mathbb{R}^{2}$ as $w_{i}(x, y)=$ $\left(L_{i}(x), F_{i}(x, y)\right), \forall i \in \mathbb{N}_{N-1}$ to construct an IFS for the given interpolation data as $\mathcal{I}=\left\{K ; w_{i}, i=\right.$ $1,2, \ldots, N-1\}$.

Theorem 2.1 ([5, Barnsley]). The above IFS $\mathcal{I}$ admits a unique attractor $G$. Further, $G$ is the graph of a continuous function $f^{*}: I \rightarrow R$ such that $f^{*}\left(x_{i}\right)=y_{i}, i=1,2, \ldots, N$.

The previous function $f^{*}$ is called a fractal interpolation function (FIF), and for implicit representation of FIF, we proceed as follows:

Let $\mathcal{G}=\left\{g: I \rightarrow \mathbb{R} \mid g\right.$ is continuous and $g\left(x_{1}\right)=$ $\left.y_{1}, g\left(x_{N}\right)=y_{N}\right\}$. Then $\mathcal{G}$ is a complete metric space with respect to the metric induced by the uniform norm $\|\cdot\|_{\infty}$ on $\mathcal{C}(I)$.

Define a mapping $T: \mathcal{G} \mapsto \mathcal{G}$ by $(T g)(x)=$ $F_{i}\left(L_{i}^{-1}(x), g \circ L_{i}^{-1}(x)\right), x \in\left[x_{i}, x_{i+1}\right], i \in \mathbb{N}_{N-1}$. Now, $T$ is a contraction mapping on the metric $\operatorname{space}\left(\mathcal{G},\|\cdot\|_{\infty}\right)$, i.e.

$$
\|T g-T h\|_{\infty} \leq|\alpha|_{\infty}\|g-h\|_{\infty}, \quad g, h \in \mathcal{G},
$$

where $\alpha=\left(\alpha_{1}, \ldots, \alpha_{N-1}\right) \in(-1,1)^{N-1}$ is called a scale vector of the IFS and $|\alpha|_{\infty}=\max \left\{\left|\alpha_{i}\right|\right.$ : $i=1,2, \ldots, N-1\}<1$. By Banach fixed point theorem, $T$ possesses a unique fixed point $f^{*} \in \mathcal{G}$, such that $\left(T f^{*}\right)(x)=f^{*}(x), \forall x \in\left[x_{1}, x_{N}\right]$. From the definition of $T, f^{*}$ satisfies

$$
\begin{aligned}
& f^{*}(x)=F_{i}\left(L_{i}^{-1}(x), f^{*} \circ L_{i}^{-1}(x)\right), \\
& x \in\left[x_{i}, x_{i+1}\right], i \in \mathbb{N}_{N-1} .
\end{aligned}
$$

$\boldsymbol{\alpha}$-fractal functions. Navascués ${ }^{13}$ observed that the IFS theory can be used to generate a family of continuous functions having fractal characteristics from a prescribed continuous function $f \in \mathcal{C}(I)$. Consider a partition $\Delta=\left\{x_{1}, x_{2}, \ldots, x_{N}\right\}$ of $I$ satisfying $x_{1}<x_{2}<\cdots<x_{N}$, a base function $b$ satisfying $b \in \mathcal{C}(I), b \neq f, b\left(x_{1}\right)=f\left(x_{1}\right)$ and $b\left(x_{N}\right)=f\left(x_{N}\right)$ and $N-1$ real numbers $\alpha_{i}$ satisfying $\left|\alpha_{i}\right|<1$. Define an IFS through the maps

$$
\begin{aligned}
L_{i}(x) & =a_{i} x+d_{i}, \\
F_{i}(x, y) & =\alpha_{i} y+f \circ L_{i}(x)-\alpha_{i} b(x), \quad i \in \mathbb{N}_{N-1},
\end{aligned}
$$

where $L_{i}$ and $F_{i}$ satisfy (2.1) and (2.2), respectively. In general $b$ can be defined through a linear map $L: \mathcal{C}(I) \rightarrow \mathcal{C}(I)$ such that $b=L f, L$ is bounded with respect to supremum norm and $L f\left(x_{1}\right)=f\left(x_{1}\right)$ and $L f\left(x_{N}\right)=f\left(x_{N}\right)$. The corresponding FIF denoted by $f_{\Delta, b}^{\alpha}=f^{\alpha}$ is referred as $\alpha$ fractal function for $f$ with respect to a scale vector $\alpha$, base functions $b$ and partition $\Delta$. The function $f^{\alpha}$ is the fixed point of the operator

$$
\begin{array}{r}
T_{\Delta, b, f}^{\alpha} g(x)=f(x)+\alpha_{i}(g-b) \circ L_{i}^{-1}(x), \\
x \in I_{i}, \quad i \in \mathbb{N}_{N-1} .
\end{array}
$$

Further, the perturbation process produces a fractal operator $\mathcal{F}_{\Delta, b}^{\alpha}=\mathcal{F}^{\alpha}: \mathcal{C}(I) \rightarrow \mathcal{C}(I)$ such that $\mathcal{F}^{\alpha}(f)=f^{\alpha}$, which is a bounded linear map for $b=L f$ and $L: \mathcal{C}(I) \rightarrow \mathcal{C}(I)$ being a bounded linear map. From (2.3), the following uniform error bound between $f$ and its fractal analog can be found (see for instance Refs. 7 and 14):

$$
\left\|f^{\alpha}-f\right\|_{\infty} \leq \frac{|\alpha|_{\infty}}{1-|\alpha|_{\infty}}\|f-b\|_{\infty} .
$$

The fractal function $f^{\alpha}$ interpolates to $f$ at the nodes:

$$
f^{\alpha}\left(x_{i}\right)=f\left(x_{i}\right), \quad i \in \mathbb{N}_{N}
$$

For $\alpha=0, f^{\alpha}$ agrees with $f$. 


\subsection{Some Classical Results}

The best known results of the American mathematician Dunham Jackson are several inequalities bounding the uniform distance between a continuous (or differentiable) function and the space of trigonometric or algebraic polynomials of degree at most $n$. For instance, in the periodic case we have the following inequality! 15

Theorem 2.2. Let $f \in \mathcal{C}[-\pi, \pi]$ be periodic. If $d_{n}^{*}(f)=d\left(f, \tau_{n}\right)$, where $d\left(f, \tau_{n}\right)$ is the minimum distance between $f$ and the space

$\tau_{n}=\left\{\sum_{k=0}^{n}\left(a_{k} \cos (k x)+b_{k} \sin (k x)\right): a_{k}, b_{k} \in \mathbb{R}\right\}$,

then $d_{n}^{*}(f) \leq \omega\left(\frac{\pi}{n+1}\right)$, where $\omega(\delta)$ is the modulus of continuity of $f$.

There is an analogous result for $f \in \mathcal{C}[-1,1]$ and the space of polynomials of degree at most $n ! 15$

Let us define the Lebesgue function $\lambda_{n}$ of polynomial interpolation with respect to a partition $\Delta: a=x_{0}<x_{1}<\cdots<x_{n}=b$ of the interval $[a, b]$,

$$
\lambda_{n}(x)=\sum_{k=0}^{n}\left|\phi_{k}(x)\right|
$$

where $\phi_{k}(x)$ is the $k$ th basic Lagrange polynomial. Let $\Lambda_{n}$ be the Lebesgue constant, defined as the supremum of $\lambda_{n}(x)$ on $[a, b]$. In general, if $p_{n}$ is the interpolating polynomial and $d_{n}^{*}(f)$ is the minimum distance from $f$ to the space of polynomials of degree at most $n$, ther 16

$$
\left\|f-p_{n}\right\|_{\infty} \leq d_{n}^{*}(f)\left(1+\Lambda_{n}\right) .
$$

Thus, the Lebesgue constants (which do not depend on $f$ ) are a measure as to how far the interpolation error exceeds the minimum error $d_{n}^{*}(f)$. The constants of Lagrange increase exponentially as $n \rightarrow \infty$ if the nodes are uniformly distributed. For example, $\Lambda_{10}=29.90$ and $\Lambda_{20}=10986.03$ (see for instance Ref. (16). With equidistant nodes, even a high degree of differentiability of $f$ is not sufficient to obtain convergence. In general, Runge-type phenomena are observed at the extremes of the interval (great oscillations). When the original function can be extended into an entire function $\widehat{f}: \mathbb{C} \rightarrow \mathbb{C}$, then the convergence is guaranteed, but this is a very strong requirement!17
The Chebyshev abscissae (zeros of Chebyshev polynomials)

$$
x_{k}=\cos \left(\frac{2 k-1}{2 n} \pi\right),
$$

for $k=1,2, \ldots, n$, are among the best options to be chosen in order to obtain low values of $\Lambda_{n}$ in the interval $[-1,1]$. However, one has the following result 18

Theorem 2.3. Let $\Lambda_{n T}$ be the Lebesgue constant of the polynomial interpolation with respect to the Chebyshev abscissae. Then

$$
\frac{2}{\pi} \log (n)+0.9625<\Lambda_{n T} \leq \frac{2}{\pi} \log (n)+1,
$$

for $n=1,2, \ldots$.

As a consequence, $\Lambda_{n T}$ tends to infinity with $n$. However, the convergence is sure for Lipschitz functions on Chebyshev partitions due to the following inequality of Jackson (see Ref. 15).

Theorem 2.4. For all Lipschitz continuous functions on $[-1,1]$ with Lipschitz constant L, the minimum error is bounded as

$$
d_{n}^{*}(f) \leq \frac{\pi L}{2 n+2} .
$$

In this case, due to (2.6) and (2.7),

$$
\left\|f-p_{n}\right\|_{\infty} \leq \frac{\pi L}{2 n+2}\left(2+\frac{2}{\pi} \log (n)\right),
$$

and the uniform convergence holds.

However, in many applications the nodes cannot be chosen. For instance, the bioelectric recordings as electrocardiograms and electroencephalograms are sampled by a device with a constant step (time period), and the partitions are uniform. This is true for tabular or historic data as well. For these cases the interpolants of Jackson type give uniform convergence with the hypotheses of continuity and periodicity (see Sec. 31).

Let $\mathcal{L}_{n}$ be the operator that maps $f$ into the $n$th Lagrange polynomial. The formula

$$
\mathcal{L}_{n} f(x)=\sum_{k=0}^{n} f\left(x_{k}\right) \phi_{k}(x)
$$

implies that

$$
\left\|\mathcal{L}_{n} f\right\|_{\infty} \leq \Lambda_{n}\|f\|_{\infty}
$$

and

$$
\left\|\mathcal{L}_{n} f-\mathcal{L}_{n} f^{\prime}\right\|_{\infty} \leq \Lambda_{n}\left\|f-f^{\prime}\right\|_{\infty} .
$$

The Lebesgue constant is equal to one in the Jackson case, and the approximation does not amplify 
the distances. Consequently, (2.6) assures a good uniform norm approximation.

Theorem 2.5. If $f \in \mathcal{C}^{2}[-\pi, \pi]$ is periodic, then

$$
\left|a_{k}\right|=\mathcal{O}\left(\frac{1}{k^{2}}\right), \quad\left|b_{k}\right|=\mathcal{O}\left(\frac{1}{k^{2}}\right)
$$

and

$$
\left\|f-S_{n}\right\|_{\infty}=\mathcal{O}\left(n^{-1}\right),
$$

where $S_{n}$ is the $n$th Fourier sum of $f$, and $a_{k}, b_{k}$ are its coefficients.

Theorem 2.6. If $f \in \mathcal{C}[-\pi, \pi]$ is periodic and satisfies a Dini-Lipschitz condition $\left[\lim _{\delta \rightarrow 0} \log (\delta) \times\right.$ $\omega(\delta)=0]$, then its Fourier series converges uniformly to $f$.

This result is achieved by the Jackson approximants with the single hypothesis of continuity (Sec. 3), and let us remember that uniform convergence implies convergence in the mean of order $p$ ( $\mathcal{L}^{p}$-norm) on compact intervals for $1 \leq p \leq \infty$.

Fractal trigonometric polynomials ${ }^{14}$ Let $\tau_{n}$ be the set of trigonometric polynomials of degree at most $n$, linearly spanned by the set

$$
\begin{gathered}
\{1, \sin (x), \cos (x), \sin (2 x), \cos (2 x), \\
\ldots, \sin (n x), \cos (n x)\} .
\end{gathered}
$$

Let $\Delta:-\pi=x_{0}<x_{1}<\cdots<x_{N}=\pi$ be a partition of the interval $[-\pi, \pi]$ and let $\mathcal{F}^{\alpha}$ be the $\alpha$-fractal operator that maps $f$ to $f^{\alpha}$ (see Sec. 2.1).

Definition 2.1. $\tau_{n}^{\alpha}=\mathcal{F}^{\alpha}\left(\tau_{n}\right)$ is the set of all $\alpha$-fractal trigonometric polynomials of degree at most $n$.

Due to the linearity of $\mathcal{F}^{\alpha}, \tau_{n}^{\alpha}$ is spanned by the set $\left\{1, \sin ^{\alpha}(x), \cos ^{\alpha}(x), \ldots, \sin ^{\alpha}(n x), \cos ^{\alpha}(n x)\right\}$, where $\mathcal{F}^{\alpha}(\sin (j x))=\sin ^{\alpha}(j x)$ and $\mathcal{F}^{\alpha}(\cos (j x))=$ $\cos ^{\alpha}(j x)$. Thus, it is clear that $\operatorname{dim} \tau_{n}^{\alpha}<+\infty$. This fact allows the existence of a finite uniform distance from $f \in \mathcal{C}[-\pi, \pi]$ to $\tau_{n}^{\alpha}$. Let $S_{n}^{\alpha}=\mathcal{F}^{\alpha} \circ S_{n}$ be the operator such that

$$
S_{n}^{\alpha}(f)=\mathcal{F}^{\alpha}\left(S_{n}(f)\right)
$$

( $\alpha$-fractal finite Fourier sum of $f$ ). $S_{n}^{\alpha}$ is a bounded linear operator. In this way Theorem[2.6 can be generalized to the fractal series (see Ref. 14 for details).

Theorem 2.7. Let $f \in \mathcal{C}[-\pi, \pi]$ be periodic and satisfying a Dini-Lipschitz condition $\left[\lim _{\delta \rightarrow 0} \log (\delta) \omega(\delta)=0\right]$. If $\left\{\alpha^{n}\right\}$ is a sequence of scale vectors such that $\alpha^{n} \rightarrow \mathbf{0}$ as $n \rightarrow \infty$, then the $\alpha$-fractal Fourier series of $f$ converges uniformly to $f$.

If one wants to use Fourier-type functions, one can take a Césaro mean. According to Féjer's theorem, the average defined as

$$
\sigma_{n}=\frac{1}{n}\left(S_{0}+S_{1}+\cdots+S_{n-1}\right)
$$

converges uniformly to $f \underline{15}$

The advantage of the Jackson approximants is the fact of being explicit in terms of the sampled values of the original function and their good properties of convergence. We will study the fractal versions of generalized discrete Jackson approximants in the following sections.

\section{FRACTAL DISCRETE GENERALIZED JACKSON APPROXIMANTS}

First, we generalize the discrete Jackson approximants with a positive exponent $\gamma>0$ in the kernel function as follows: Define

$$
D_{m, \gamma}(f)(x)=H_{m, \gamma} \sum_{i=1}^{2 m} f\left(x_{i}\right) P_{m, i, \gamma}(x),
$$

where

$$
\begin{aligned}
P_{m, i, \gamma}(x) & =\left|\frac{\sin \left(\frac{m\left(x_{i}-x\right)}{2}\right)}{m \sin \left(\frac{x_{i}-x}{2}\right)}\right|^{\gamma}, \\
H_{m, \gamma}^{-1} & =\sum_{i=1}^{2 m} P_{m, i, \gamma}(x),
\end{aligned}
$$

and

$$
x_{i+1}-x_{i}=\frac{\pi}{m} \quad \text { for } i=1,2, \ldots, 2 m-1 .
$$

The discrete Jackson approximants were defined for a fixed exponent $\gamma=4$ by (3.1) (see p. 456 of Ref. 19). For a positive exponent $\gamma>0, H_{m, \gamma}=$ $H_{m, \gamma}(x)$ depends on the variable $x$ although we will preserve the original notation omitting it. Note that $H_{m, 4}$ is a constant. However, the value $\gamma=4$ is not always the optimal choice, as shown in Table 1.

Now, we will define fractal versions of discrete Jackson approximants by using fractal analogs of the kernel function $P_{m, i, \gamma}(x)$. For this accomplished goal, the basis function $P_{m, i, \gamma}(x)$ is perturbed using suitable base functions $b_{m, i, \gamma}$, suitable scale vector $\alpha$ and partition of $[-\pi, \pi]$ in order to obtain its fractal analogs $P_{m, i, \gamma}^{\alpha}(x)$. If we have $N$ interpolation 
Table 1 Point-Wise Errors Between $f(x)$ and $D_{m, \gamma}(x)$ for Different Choices of $m, f$ and $\gamma$.

\begin{tabular}{lccc}
\hline & $\gamma=\mathbf{4}$ & $\gamma=\mathbf{4 . 5}$ & $\gamma=\mathbf{5}$ \\
\hline$m=12,(\sin (\pi / 5) \cos (\pi / 5))^{3}$ & 0.00948607 & 0.00948607 & 0.00958445 \\
$m=12,(\sin (\pi / 7) \cos (\pi / 7))^{3}$ & 0.00278478 & 0.00278478 & 0.00368186 \\
$m=5, \sqrt{\sin (\pi / 3)}$ & 0.03208200 & 0.02814760 & 0.02471950 \\
$m=6, \sqrt{\sin (\pi / 4)}$ & 0.02640850 & 0.02458460 & 0.02352260 \\
$m=5, \log (2+\cos (\pi / 3))$ & 0.01834100 & 0.01787330 & 0.01861590 \\
$m=6, \log (2+\cos (\pi / 4))$ & 0.01377970 & 0.01252320 & 0.01191420 \\
\hline
\end{tabular}

points in $[-\pi, \pi]$, then $\alpha=\left(\alpha_{1}, \alpha_{2}, \ldots, \alpha_{N-1}\right) \in$ $(-1,1)^{N-1}$, and $N$ is independent of $m$. Thus, the fractal function associated to the generalized discrete Jackson approximants is defined as

$$
D_{m, \gamma}^{\alpha}(f)(x)=H_{m, \gamma} \sum_{i=1}^{2 m} f\left(x_{i}\right) P_{m, i, \gamma}^{\alpha}(x) .
$$

Example 3.1 (Fractal Jackson basis function). Consider $m=10$ and $\gamma=4$. The classical third Jackson basis function $P_{10,3,4}$ is plotted in Fig. 1h over $[-\pi, \pi]$. To construct fractal Jackson basis function over the interval, we have taken a partition with 10 equidistant subintervals of $[-\pi, \pi]$, i.e. $N=11$. The base function $b_{10,3,4}$ is taken as a straight line joining the end points to construct the fractal basis function $P_{10.3 .4}^{\alpha}(x)$ with different scales $\alpha$. First, we choose a scale vector, where each component is positive as $\alpha=(0.4,0.1$, $0.1,0,0,0,0,0.1,0.1,0.2)$. The corresponding third fractal Jackson basis function generated in Fig. 1 b is irregular and nonnegative. Next, the scale vector contains some negative components as $\alpha=$ $(0.3,0,0,0,0,0,0,0,-0.4,0.4)$, and the corresponding fractal Jackson basis function is plotted in Fig. 17. For a smoother version of fractal Jackson basis function, the magnitude of components of $\alpha$ are reduced as $\alpha=(0.05,-0.06,0.1$, $-0.04,0.08,-0.04,0.14,-0.04,0.04,-0.04)$ to generate Fig. 11. The range of $P_{m, i, \gamma}^{\alpha}(x)$ can be restricted based on the suitable choice $\alpha$ (see Ref. 8 for details). It is observed that based on the choice of $\alpha$, we can get fractal smooth or nonsmooth Jackson basis functions to approximate periodic smooth or nonsmooth signals that are known at discrete points.

In order to prove the main approximation result by the fractal version of the generalized discrete
Jackson operator in Theorem 3.1 we need the following lemmas.

Lemma 3.1. For all $m=1,2, \ldots, \gamma>0$, and $v \in R$

$$
\left|\frac{\sin (m v)}{m \sin (v)}\right|^{\gamma} \leq 1
$$

Proof. For $\gamma=2$, it is known that (see p. 340 of Ref. 12)

$$
\begin{aligned}
\left(\frac{\sin \left(\frac{n x}{2}\right)}{\sin \left(\frac{x}{2}\right)}\right)^{2}= & n+2[(n-1) \cos x+(n-2) \\
& \times \cos 2 x+\cdots+\cos ((n-1) x)],
\end{aligned}
$$

for $n \in \mathbb{N}$ and $x \in \mathbb{R}$. Taking $n=m$ and $x=2 v$ in the above identity, we have

$$
\begin{aligned}
\left(\frac{\sin (m v)}{\sin (v)}\right)^{2}= & m+2[(m-1) \cos (2 v)+(m-2) \\
& \times \cos (4 v)+\cdots+\cos (2(m-1) v)] .
\end{aligned}
$$

Then

$$
\begin{aligned}
& \left|\frac{\sin (m v)}{m \sin (v)}\right|^{\gamma} \\
& \quad=\frac{1}{m^{\gamma}} \mid m+2((m-1) \cos (2 v)+(m-2) \\
& \quad \times \cos (4 v)+\cdots+\cos (2(m-1) v))\left.\right|^{\gamma / 2} \\
& \quad \leq \frac{1}{m^{\gamma}}\left(m+2\left(\frac{1+(m-1)}{2}\right)(m-1)\right)^{\gamma / 2} \\
& \quad \leq \frac{1}{m^{\gamma}}\left(m^{2}\right)^{\gamma / 2}=1 .
\end{aligned}
$$

Lemma 3.2. For $v \in[0, \pi / 2]$,

$$
\sin (v) \geq \frac{2 v}{\pi}
$$




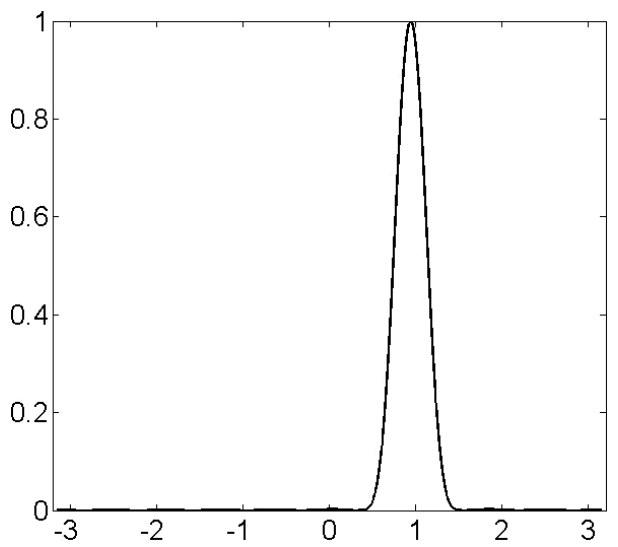

(a) $P_{10,3,4}(x)$ when $\alpha=\mathbf{0}$

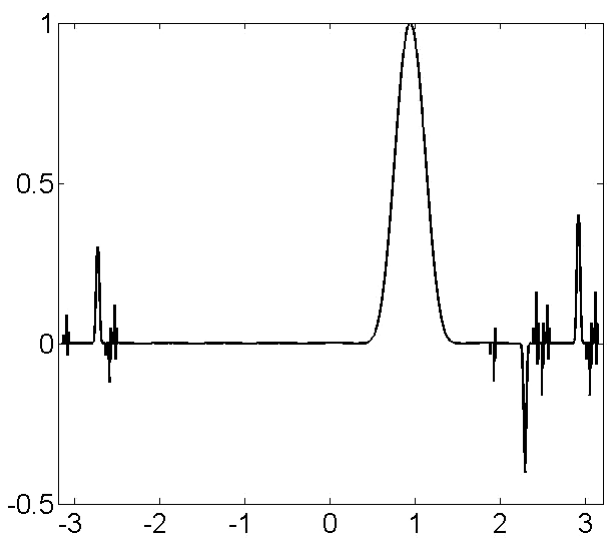

(c) $P_{10,3,4}^{\alpha}(x)$ with $\alpha=(0.3,0,0,0,0,0,0,0,-0.4,0.4)$

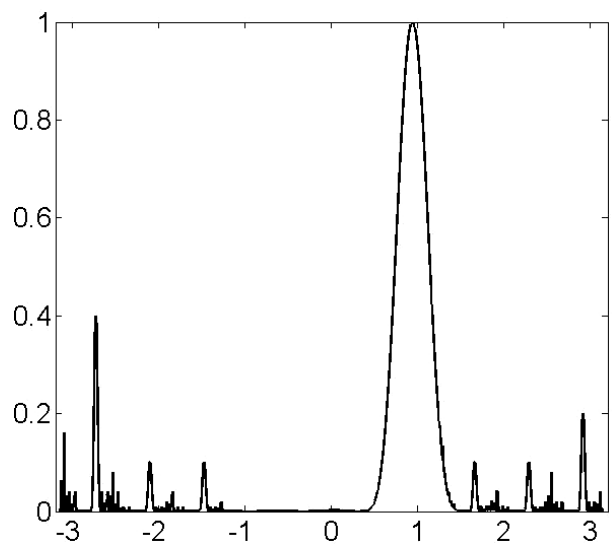

(b) $P_{10,3,4}^{\alpha}(x)$ with $\alpha=(0.4,0.1,0.1,0,0,0,0,0.1,0.1,0.2)$

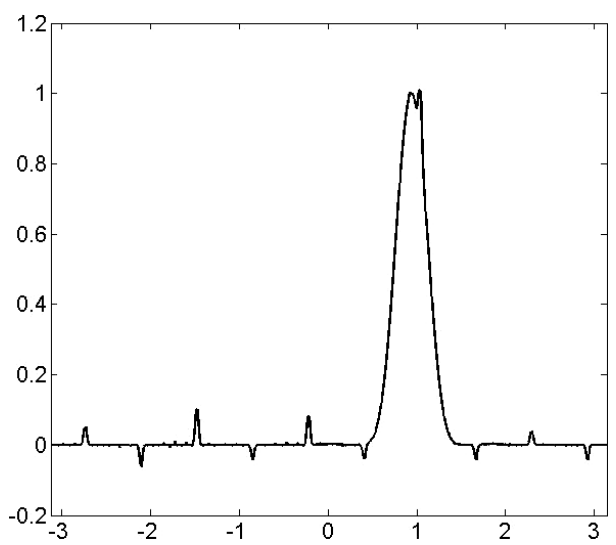

(d) $P_{10,3,4}^{\alpha}(x)$ with $\alpha=(0.05,-0.06,0.1$, $-0.04,0.08,-0.04,0.14,-0.04,0.04,-0.04)$

Fig. 1 Basis functions of generalized Jackson approximation.

Proof. The function $\sin (v)$ is concave in the interval $[0, \pi / 2]$ and thus

$$
\sin (v) \geq r(v)
$$

where $r(v)$ is the line joining $(0,0)$ and $(\pi / 2,1)$. But $r(v)=2 v / \pi$, and hence the result.

Theorem 3.1. Let $f \in \mathcal{C}[-\pi, \pi]$ be Hölder continuous such that for $x, x^{\prime} \in[-\pi, \pi]$,

$$
\left|f(x)-f\left(x^{\prime}\right)\right| \leq K\left|x-x^{\prime}\right|^{q},
$$

for some $q$ such that $0<q \leq 1$. Then for $\gamma>q+1$,

$$
\begin{aligned}
& \left\|D_{m, \gamma}^{\alpha}(f)-f\right\|_{\infty} \\
& \leq \frac{K}{2}\left(\frac{\pi}{2}\right)^{\gamma}\left(\frac{\pi}{2 m}\right)^{q} \\
& \quad \times\left(1+2^{q}+2^{\gamma}\left(\frac{1}{\gamma-(q+1)}+\frac{1}{\gamma-1}\right)\right) \\
& \quad+m\left(\frac{\pi}{2}\right)^{\gamma}\|f\|_{\infty} \frac{|\alpha|_{\infty}}{1-|\alpha|_{\infty}},
\end{aligned}
$$

where $\alpha$ is a suitable scaling vector used to construct the fractal perturbation of $P_{m, i, \gamma}$ based on a partition of $[-\pi, \pi]$.

Proof. For the required upper bound, we will use

$$
\begin{aligned}
& \left\|D_{m, \gamma}^{\alpha}(f)-f\right\|_{\infty} \\
& \quad \leq\left\|D_{m, \gamma}^{\alpha}(f)-D_{m, \gamma}(f)\right\|_{\infty}+\left\|D_{m, \gamma}(f)-f\right\|_{\infty} .
\end{aligned}
$$

Now using the definitions of $D_{m, \gamma}$ (3.1) and $H_{m, \gamma}$ (3.2), and changing $x_{i}=x+2 u_{i}$, we obtain

$$
\begin{aligned}
& \left|E_{m, \gamma}(f)(x)\right| \\
& \quad:=\left|D_{m, \gamma}(f)(x)-f(x)\right| \\
& \quad \leq H_{m, \gamma} \sum_{i=1}^{2 m}\left|f\left(x+2 u_{i}\right)-f(x)\right| P_{m, i, \gamma}(x) .
\end{aligned}
$$


Using the Lipschitz constant and exponent,

$\left|E_{m, \gamma}(f)(x)\right| \leq H_{m, \gamma} K 2^{q} \sum_{i=1}^{2 m}\left|u_{i}\right|^{q} \mid P_{m, i, \gamma}\left(x_{i}-2 u_{i}\right)$.

Since $P_{m, i, \gamma}$ is periodic, we can assume $u_{i} \in$ $[-\pi / 2, \pi / 2]$. Considering increasing order in $\left|u_{i}\right|$ and denoting them by $v_{0}, v_{1}, \ldots, v_{2 m-1}$,

$$
\left|E_{m, \gamma}(f)(x)\right| \leq H_{m, \gamma} K 2^{q} \sum_{i=0}^{2 m-1} v_{i}^{q} P_{m, i, \gamma}\left(x_{i}-2 v_{i}\right),
$$

where

$$
\frac{\pi i}{4 m} \leq v_{i} \leq \frac{\pi(i+1)}{4 m} \leq \frac{\pi}{2},
$$

for $i=0,1, \ldots, 2 m-1$ (see p. 458 of Ref. 19)). Here $P_{m, i, \gamma}\left(x_{i}-2 v_{i}\right)=\left|\frac{\sin \left(m v_{i}\right)}{m \sin \left(v_{i}\right)}\right|^{\gamma}$. Now from Lemma3.1,

$$
\left|\frac{\sin \left(m v_{i}\right)}{m \sin \left(v_{i}\right)}\right|^{\gamma} \leq 1 \quad \text { if } i=0,1 .
$$

For $i \geq 2$, using Lemma 3.2 ,

$$
m \sin \left(v_{i}\right) \geq m \sin \left(\frac{\pi i}{4 m}\right) \geq m 2 \frac{i}{4 m} \geq \frac{i}{2} .
$$

As a consequence, for $i \geq 2$,

$$
\left|\frac{\sin \left(m v_{i}\right)}{m \sin \left(v_{i}\right)}\right|^{\gamma} \leq\left(\frac{2}{i}\right)^{\gamma} \text {. }
$$

Bearing in mind (3.9) for $i=0,1$ and (3.10) for $i \geq 2$, we obtain

$$
\begin{aligned}
& \left|E_{m, \gamma}(f)(x)\right| \\
& \leq H_{m, \gamma} K 2^{q} \sum_{i=0}^{2 m-1} v_{i}^{q}\left|\frac{\sin \left(m v_{i}\right)}{m \sin \left(v_{i}\right)}\right|^{\gamma} \\
& \leq H_{m, \gamma} K 2^{q}\left[v_{0}^{q}+v_{1}^{q}+\sum_{i=2}^{2 m-1} v_{i}^{q}\left(\frac{2}{i}\right)^{\gamma}\right] .
\end{aligned}
$$

By definition of $v_{i}$,

$$
v_{0}^{q} \leq\left(\frac{\pi}{4 m}\right)^{q}, \quad v_{1}^{q} \leq\left(\frac{\pi}{2 m}\right)^{q},
$$

and

$$
\begin{aligned}
\sum_{i=2}^{2 m-1} v_{i}^{q}\left(\frac{2}{i}\right)^{\gamma} & \leq 2^{\gamma}\left(\frac{\pi}{4 m}\right)^{q} \sum_{i=2}^{2 m-1}(i+1)^{q} \frac{1}{i^{\gamma}} \\
& =2^{\gamma}\left(\frac{\pi}{4 m}\right)^{q} \sum_{i=2}^{2 m-1}\left(\frac{1}{i^{\gamma-q}}+\frac{1}{i^{\gamma}}\right) .
\end{aligned}
$$

The last sum is part of lower Riemann sums of the functions $1 / x^{\gamma-q}$ and $1 / x^{\gamma}$, in the interval $[1,+\infty)$ with unit step, respectively:

$$
\begin{aligned}
\sum_{i=2}^{2 m-1} \frac{1}{i^{\gamma-q}} \leq \int_{1}^{\infty} \frac{d x}{x^{\gamma-q}}= & \frac{1}{\gamma-(q+1)} \\
& \text { if } \gamma>q+1 .
\end{aligned}
$$

Likewise

$$
\sum_{i=2}^{2 m-1} \frac{1}{i^{\gamma}} \leq \int_{1}^{\infty} \frac{d x}{x^{\gamma}}=\frac{1}{\gamma-1}, \quad \text { if } \gamma>1 .
$$

Collecting the above results, (3.12) and 3.13 in (3.11), we have

$$
\begin{aligned}
& \left|E_{m, \gamma}(f)(x)\right| \\
& \leq H_{m, \gamma} K 2^{q}\left[\left(\frac{\pi}{4 m}\right)^{q}+\left(\frac{\pi}{2 m}\right)^{q}\right. \\
& \left.\quad+2^{\gamma}\left(\frac{\pi}{4 m}\right)^{q}\left(\frac{1}{\gamma-(q+1)}+\frac{1}{\gamma-1}\right)\right] .
\end{aligned}
$$

Let us bound now $H_{m, \gamma}$ which is defined in (3.2).

$$
\begin{aligned}
H_{m, \gamma}^{-1}= & \sum_{i=0}^{2 m-1}\left|\frac{\sin \left(m v_{i}\right)}{m \sin \left(v_{i}\right)}\right|^{\gamma}>\left|\frac{\sin \left(m v_{0}\right)}{m \sin \left(v_{0}\right)}\right|^{\gamma} \\
& +\left|\frac{\sin \left(m v_{1}\right)}{m \sin \left(v_{1}\right)}\right|^{\gamma} .
\end{aligned}
$$

Since $m v_{i} \leq \pi / 2$ for $i=0,1$, from (3.8) and Lemma 3.2,

$$
\frac{\sin \left(m v_{i}\right)}{m \sin \left(v_{i}\right)} \geq \frac{2 m v_{i}}{m \pi \sin \left(v_{i}\right)} \geq \frac{2}{\pi},
$$

and thus from (3.15),

$$
H_{m, \gamma}^{-1}>2\left(\frac{2}{\pi}\right)^{\gamma} \text {. }
$$

Now for the first term of right-hand side of (3.5),

$$
\begin{aligned}
& \left|D_{m, \gamma}^{\alpha}(f)(x)-D_{m, \gamma}(f)(x)\right| \\
& \quad=\left|H_{m, \gamma} \sum_{i=1}^{2 m} f\left(x_{i}\right)\left(P_{m, i, \gamma}^{\alpha}(x)-P_{m, i, \gamma}(x)\right)\right| \\
& \quad \leq H_{m, \gamma} \sum_{i=1}^{2 m}\left|f\left(x_{i}\right)\right|\left|P_{m, i, \gamma}^{\alpha}(x)-P_{m, i, \gamma}(x)\right|
\end{aligned}
$$




$$
\begin{aligned}
\leq & H_{m, \gamma}\|f\|_{\infty} \sum_{i=1}^{2 m}\left|P_{m, i, \gamma}^{\alpha}(x)-P_{m, i, \gamma}(x)\right| \\
\leq & \left(\frac{\pi}{2}\right)^{\gamma} m\|f\|_{\infty} \max _{1 \leq i \leq 2 m}\left\|P_{m, i, \gamma}^{\alpha}-P_{m, i, \gamma}\right\|_{\infty} \\
\leq & \left(\frac{\pi}{2}\right)^{\gamma} m\|f\|_{\infty} \frac{|\alpha|_{\infty}}{1-|\alpha|_{\infty}} \\
& \times \max _{1 \leq i \leq 2 m}\left\|P_{m, i, \gamma}-b_{m, i, \gamma}\right\|_{\infty}
\end{aligned}
$$

due to (3.16) and 2.4. The maps $b_{m, i, \gamma}$ are used to define the fractal functions $P_{m, i, \gamma}^{\alpha}$. Let us bear in mind now that, due to Lemma 3.1, the kernel functions $P_{m, i, \gamma}$ are bounded by one, i.e. $0 \leq P_{m, i, \gamma}(x) \leq$ $1, \forall x \in[-\pi, \pi]$. The maps $b_{m, i, \gamma}$ can be chosen such that they are bounded by one (taking for instance the lines joining the extremes of the graph of $\left.P_{m, i, \gamma}\right)$.
Consequently, we have $\left|P_{m, i, \gamma}(x)-b_{m, i, \gamma}(x)\right| \leq 1$ for all $i=1,2, \ldots 2 m, x \in[-\pi, \pi]$. Finally, using (3.5), (3.14) and (3.17), the proof follows.

Corollary 3.1. If $f \in \mathcal{C}[-\pi, \pi]$ is Hölder continuous with exponent $q(0<q \leq 1)$ and $\gamma>q+1$ and if we select scaling factors $\alpha$ such that $\left|\alpha_{i}\right|=$ $\mathcal{O}\left(m^{-(q+1)}\right)$, then $D_{m, \gamma}^{\alpha}(f)$ converges uniformly to $f$ as $m$ tends to infinity. The order of convergence $\mathcal{O}\left(m^{-(q+1)}\right)$ does not depend on $\gamma$.

Example 3.2. In order to show the versatility and diversity associated with fractal version of the generalized discrete Jackson approximants, we consider a periodic function on $[-\pi, \pi]$ as $f(x)=\sin ^{3} x+$ $2 \cos ^{2} x$ as in Fig. 2a. Take $m=6$, for which 12 values of $f$ are taken for reconstruction of approximants in our method. First, we plot the generalized

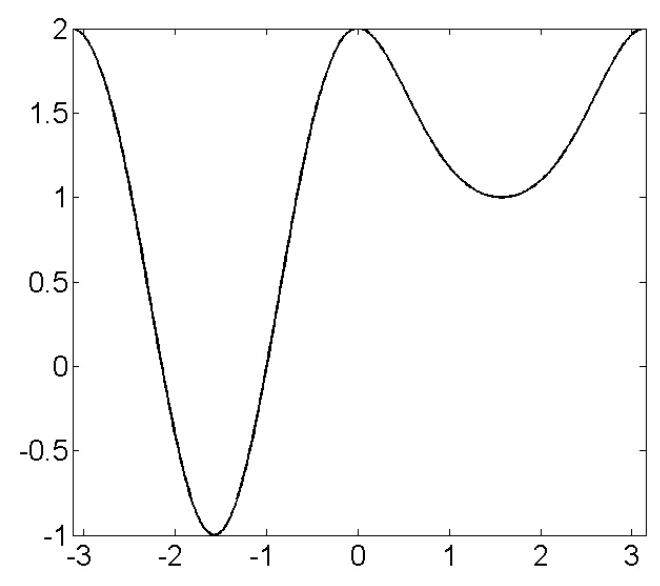

(a) Graph of the function $f(x)=\sin ^{3} x+2 \cos ^{2} x$

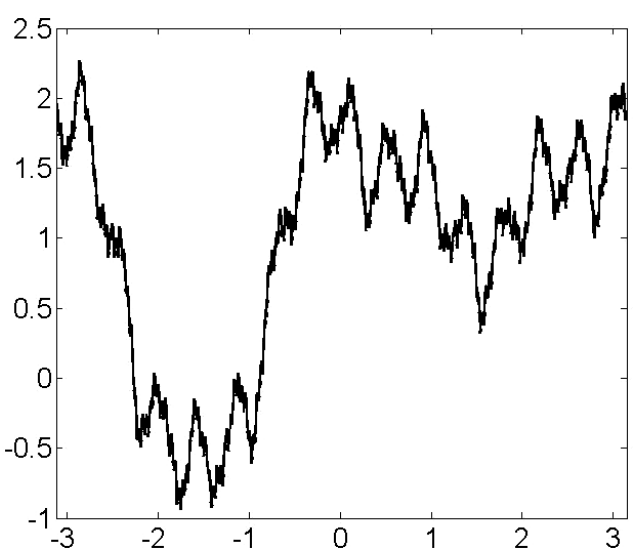

(c) $D_{6,5}^{\alpha}(f)$ with $\alpha=(0.2,-0.2,0.2,-0.2$, $0.2,-0.2,0.2,-0.2,0.2,-0.2)$

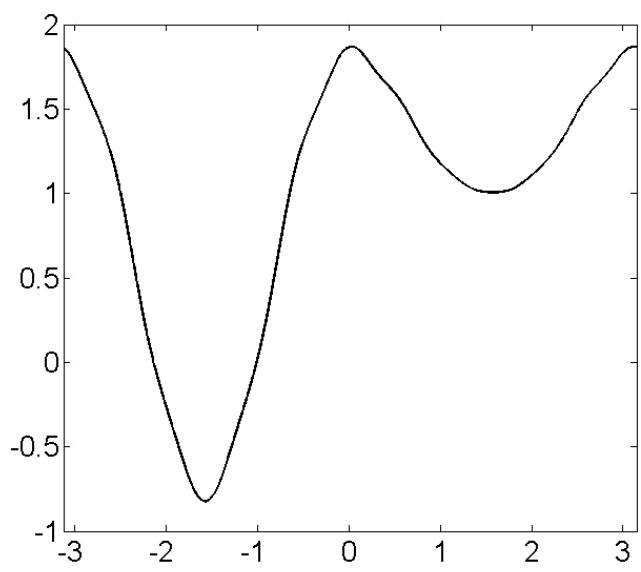

(b) Generalized Jackson approximant

$$
D_{6,5}(f)(x)
$$

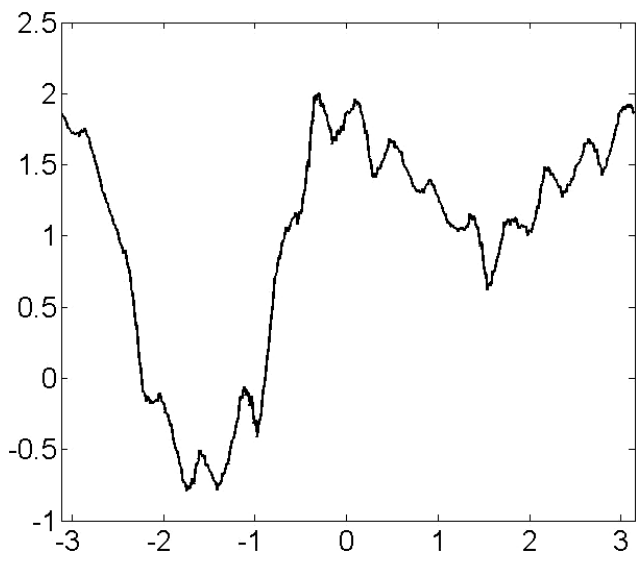

(d) $D_{6,5}^{\alpha}(f)$ with $\alpha=(0.05,-0.1,0.1,-0.15$, $0.15,-0.1,0,0.05,-0.12,0.09,-0.08)$

Fig. 2 Original function and fractal versions of its generalized Jackson discrete approximants. 
discrete Jackson approximant $D_{6,5}(f)$ in Fig. $2 \mathrm{~b}$ with exponent $\gamma=5$. In order to get the fractal version of the Jackson approximant, consider an equidistant partition of $[-\pi, \pi]$ with $N=11$. We have taken two different scaling vectors in $(-1,1)^{10}$ to obtain fractal version of Jackson approximants in Figs. 2r and 2d. Note that these 12 sample points are present in our proposed Jackson approximants whose shapes are very much different. In practice, there is no need to believe that sample points are being generated only from a smooth function. According to Corollary 3.1, when the sample size increases, the fractal Jackson approximant converges to the original function with proper choice of scaling factors. Thus, a priori hypothesis on nature of $f$ may help us to choose a smooth or nonsmooth periodic approximant of $f$ in our methodology.

From here on we consider a general continuous function $f \in \mathcal{C}[-\pi, \pi]$, consequently uniformly continuous in this interval. Let us remind the modulus of continuity $\omega$ of $f$.

Definition 3.1. Let $f(x)$ be a uniformly continuous function in a compact interval $I$. The modulus of continuity of $f$ is defined as

$$
\begin{aligned}
\omega(\delta) & =\omega(\delta ; f) \\
& =\sup _{\left|x-x^{\prime}\right| \leq \delta}\left|f(x)-f\left(x^{\prime}\right)\right|, \quad x, x^{\prime} \in I .
\end{aligned}
$$

Some of the properties of the modulus of continuity are (see for instance Refs. 20] and 21):

- If $\delta \leq \delta^{\prime}$ then $\omega(\delta) \leq \omega\left(\delta^{\prime}\right)$.

- $\lim _{\delta \rightarrow 0} \omega(\delta)=0$.

- $\omega(m \delta) \leq m \omega(\delta)$ for $m \in \mathbb{N}$.

- $\omega(\lambda \delta) \leq(\lambda+1) \omega(\delta)$ for $\lambda \geq 0$.

Let us recall the Riemann zeta function

$$
\zeta(s)=\sum_{n=1}^{\infty} \frac{1}{n^{s}} .
$$

Theorem 3.2. If $f \in \mathcal{C}[-\pi, \pi]$ and $\gamma>2$,

$$
\begin{aligned}
\left\|D_{m, \gamma}^{\alpha}(f)-f\right\|_{\infty} \leq & \left(\frac{\pi}{2}\right)^{\gamma} \omega\left(\frac{\pi}{4 m}\right)\left(1+2^{\gamma} \zeta(\gamma-1)\right) \\
& +m\left(\frac{\pi}{2}\right)^{\gamma}\|f\|_{\infty} \frac{|\alpha|_{\infty}}{1-|\alpha|_{\infty}}
\end{aligned}
$$

where $\alpha$ is a suitable scaling vector used to construct the fractal perturbation of the kernel functions $P_{m, i, \gamma}$.

Proof. In this case, considering the properties of $\omega,(3.6)$ is transformed to

$$
\left|E_{m, \gamma}(f)(x)\right| \leq H_{m, \gamma} 2 \sum_{i=0}^{2 m-1} \omega\left(v_{i}\right)\left|\frac{\sin \left(m v_{i}\right)}{m \sin \left(v_{i}\right)}\right|^{\gamma} .
$$

Using (3.8), we have

$$
\omega\left(v_{i}\right) \leq \omega\left(\frac{\pi(i+1)}{4 m}\right) \leq(i+1) \omega\left(\frac{\pi}{4 m}\right),
$$

and thus, bearing in mind (3.9) and (3.10),

$$
\begin{aligned}
& \left|E_{m, \gamma}(f)(x)\right| \\
& \leq 2 H_{m, \gamma} \omega\left(\frac{\pi}{4 m}\right) \sum_{i=0}^{2 m-1}(i+1)\left|\frac{\sin \left(m v_{i}\right)}{m \sin \left(v_{i}\right)}\right|^{\gamma} \\
& \quad=2 H_{m, \gamma} \omega\left(\frac{\pi}{4 m}\right)\left(1+\sum_{i=1}^{2 m} i\left(\frac{2}{i}\right)^{\gamma}\right) .
\end{aligned}
$$

Consequently,

$$
\left|E_{m, \gamma}(f)(x)\right| \leq 2 H_{m, \gamma} \omega\left(\frac{\pi}{4 m}\right)\left(1+2^{\gamma} \zeta(\gamma-1)\right) .
$$

The fractal part is similar to that of the previous theorem.

Corollary 3.2. If $f \in \mathcal{C}[-\pi, \pi], \gamma>2$ and if we choose scaling factors $\alpha$ such that $m|\alpha|_{\infty}$ owns a rate of convergence similar to that of $\omega$, then $D_{m, \gamma}^{\alpha}(f)$ converges to $f$ uniformly as $m$ tends to infinity. The order of convergence does not depend on $\gamma$.

Remark 3.1. Theorem 3.1 cannot be deduced from Theorem 3.2 because the interval of values of $\gamma$ is different. The range of convergence values of $\gamma$ is wider in Theorem $3.1[\gamma>q+1]$ with the stronger assumption on $f$. By Theorem [3.2, the hypothesis of continuity alone is sufficient for a similar result when $\gamma>2$. The error bounds obtained in these theorems ensure the convergence of the fractal approximant. In practice, if the signal is periodic and irregular (for instance, a nonsmooth variable defined on the circle), the proposed fractal models 
may be more suitable than the standard trigonometric functions.

Corollary 3.3. If $f \in \mathcal{C}[-\pi, \pi]$ admits a bounded first derivative and $\gamma>2$, then

$$
\begin{aligned}
& \left\|D_{m, \gamma}^{\alpha}(f)-f\right\|_{\infty} \\
& \leq\left(\frac{\pi}{2}\right)^{\gamma}\left(\frac{\pi}{4 m}\right)\left\|f^{\prime}\right\|_{\infty}\left(1+2^{\gamma} \zeta(\gamma-1)\right) \\
& \quad+m\left(\frac{\pi}{2}\right)^{\gamma}\|f\|_{\infty} \frac{|\alpha|_{\infty}}{1-|\alpha|_{\infty}},
\end{aligned}
$$

where $\alpha$ is a suitable scaling vector used to construct the fractal perturbation of the kernel functions $P_{m, i, \gamma}$.

Proof. In this case, the modulus of continuity $\omega$ satisfies

$$
\omega(\delta) \leq \delta\left\|f^{\prime}\right\|_{\infty},
$$

and the result is a consequence of Theorem 3.2 .

Corollary 3.4. If $f$ admits a bounded derivative, $\gamma>2$ and $\left|\alpha_{i}\right| \leq \frac{1}{m^{2}}$, then the approximant converges uniformly to $f$ as $m^{-1}$ at least.

Let us consider now the periodic functions belonging to $\mathcal{L}^{p}[-\pi, \pi], p \in[1,+\infty)$, and defined at every point of the interval.

For $f \in \mathcal{L}^{p}[-\pi, \pi], p \in[1,+\infty)$, there exists the modulus of $p$-integrability (or integral modulus of continuity) of $f$

$$
\begin{aligned}
& \omega_{p}(\delta) \\
& =\sup \left\{\left(\int_{-\pi}^{\pi}|f(x+h)-f(x)|^{p} d t\right)^{1 / p} ;|h| \leq \delta\right\} .
\end{aligned}
$$

The properties of $\omega_{p}(\delta)$ are similar to those of the modulus of continuity (see for instance p. 162 of Ref.20).

Theorem 3.3. If $f \in \mathcal{L}^{p}[-\pi, \pi], p \in[1,+\infty)$ and $\gamma>2$, then

$$
\begin{aligned}
\left\|D_{m, \gamma}^{\alpha}(f)-f\right\|_{p} \leq & \left(\frac{\pi}{2}\right)^{\gamma} \omega_{p}\left(\frac{\pi}{4 m}\right)\left(1+2^{\gamma} \zeta(\gamma-1)\right) \\
& +m\left(\frac{\pi}{2}\right)^{\gamma}\|f\|_{\infty} \frac{|\alpha|_{\infty}}{1-\mid \alpha \|_{\infty}}
\end{aligned}
$$

where $\alpha$ is suitable scaling vector used to construct the fractal perturbation of the kernel functions $P_{m, i, \gamma}$.
Proof. It is left to the reader, due to similarity with previous theorem.

The statement about the convergence of the fractal interpolant is valid here, considering the new modulus $\omega_{p}$.

Remark 3.2. The convergence in $\mathcal{L}^{p}$ is implied by the uniform convergence as well $(1 \leq p<+\infty)$ but the last condition is stronger.

Example 3.3. Consider a periodic equidistant data set $\left\{\left(-\frac{4 \pi}{5},-1\right),\left(-\frac{3 \pi}{5}, 2\right),\left(-\frac{2 \pi}{5}, 3\right),\left(-\frac{\pi}{5}, 2\right)\right.$, $\left.(0,1),\left(\frac{\pi}{5}, 2\right),\left(\frac{2 \pi}{5}, 3\right),\left(\frac{3 \pi}{5}, 2\right),\left(\frac{4 \pi}{5}, 1\right),(\pi, 0)\right\}$ over the interval $I=(-\pi, \pi]$. Clearly, $m=5$ in this case, and we take $\gamma=4$ for the reconstruction of discrete Jackson approximants based on these samples. Figure $3 \mathrm{a}$ is the plot of classical discrete Jackson approximant $D_{5,4}(x)$ which does not interpolate the data of at least four points. Now, we wish to construct a fractal version of $D_{5,4}(x)$ by taking $N=11$ and different choices of scale vector. The base function is taken as the line joining the end points of the graphs. When a scale vector $\alpha=(0.2,-0.05$, $0.15,-0.15,0.05,0.15,-0.1,0.1,0,0.05)$ is chosen such that $\sum\left|\alpha_{i}\right|=1$, we obtain a fractal Jackson approximant that does not interpolate at least two points in Fig. 3b. It is clear from Fig. 3at that with smooth fractal Jackson approximants, we cannot approximate the given data set accurately. According to Theorems 3.1 and 3.2, if we take larger values of $m$, we can approximate the given data set properly. But for fractal case, the advantage is that even for small values of $m$ only, the data can be approximated accurately with the help of suitable choice of partition points and scaling vector. Again with the same choice of base functions and partition as in Fig. 3 b, we have constructed a nonsmooth fractal Jackson approximant with $\alpha=(-0.45,0.35$, $-0.15,-0.15,0.2,0.15,0.25,-0.15,0,0.5)$ in Fig. 3 s which interpolates almost all data points. In order to get a more closer approximant, a nonuniform partition of $I$ is taken as $[-\pi,-\pi / 2,-\pi / 3,-\pi / 4$, $-\pi / 5,-\pi / 6,-\pi / 7,0, \pi / 7, \pi / 6, \pi / 5, \pi / 4, \pi / 3, \pi / 2, \pi]$. In order to define the fractal function, the scale vector $\alpha$ is taken as $(0.45,-0.12,0.1,0,0.1,-0.1$, $-0.05,0.1,-0.05,0.15,-0.15,0.2,-0.15,0)$, we have the fractal version of discrete Jackson approximant $D_{5,4}^{\alpha}(x)$ shown in Fig. 3 3 which interpolates all data points very accurately. It is worth noting that in general the larger the values of components of $\alpha$, the fractal Jackson approximants are nonsmooth or irregular in nature. Thus, the proposed Jackson 


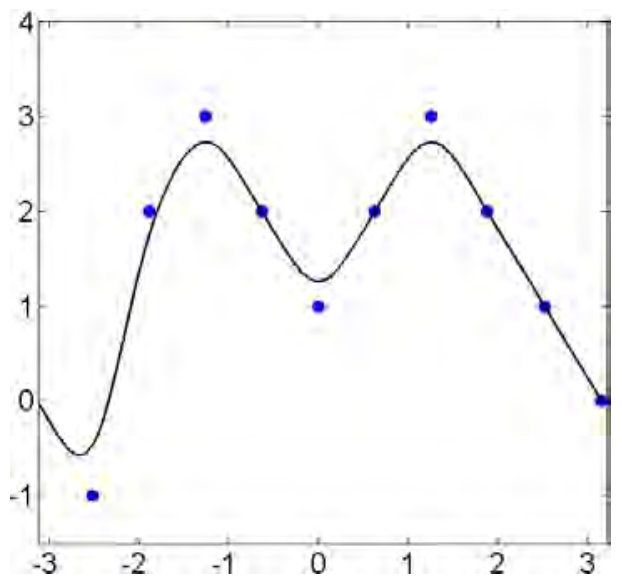

(a) Classical discrete Jackson approximant $D_{5,4}(x)$

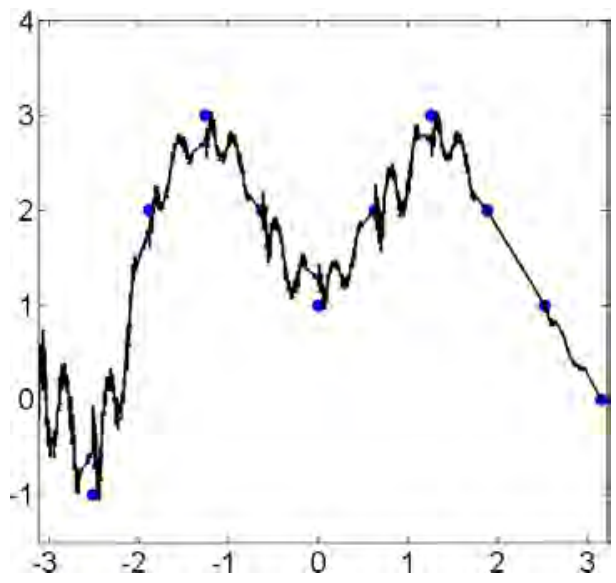

(c) Nonsmooth $D_{5,4}^{\alpha}(x)$ with 11 approximation data

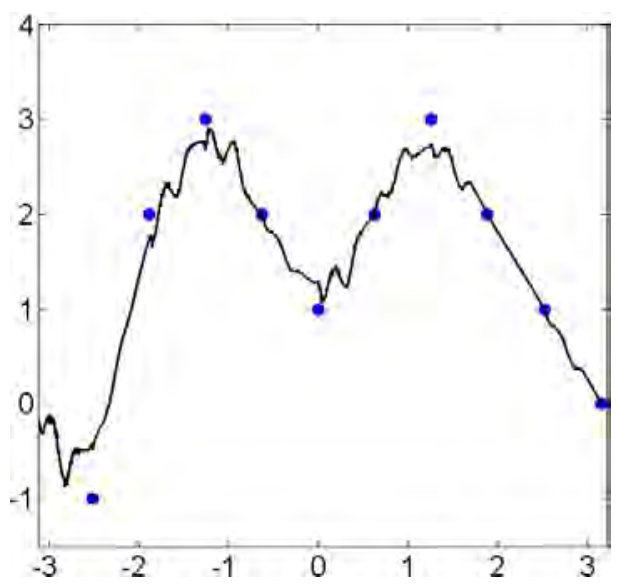

(b) $D_{5,4}^{\alpha}(x)$ with 11 approximation data

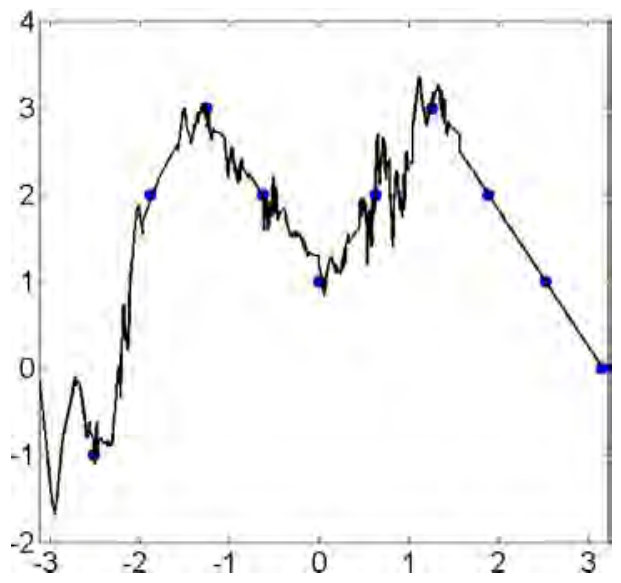

(d) Nonsmooth $D_{5,4}^{\alpha}(x)$ with 15 approximation data

Fig. 3 Fractal generalized Jackson discrete approximants of the set defined in Example 3.3

approximant combines the advantages of both traditional and fractal methodologies.

\section{PROPERTIES AND BOUNDS OF $D_{m, \gamma}^{\alpha}$}

Let us define, for all $x \in[-\pi, \pi]$,

$$
h_{m, i, \gamma}^{\alpha}(x)=H_{m, \gamma} P_{m, i, \gamma}^{\alpha}(x) .
$$

$\left\{h_{m, i, \gamma}^{\alpha}\right\}$ are continuous functions because $P_{m, i, \gamma}^{\alpha}$ are and the denominator $H_{m, \gamma}^{-1}$ is not null for every $x \in$ $I$ due to (3.16).

For $\gamma>2$ and $m \in N$ fixed, let us define

$$
\mathcal{H}_{m, \gamma}^{\alpha}=\operatorname{span}\left(h_{m, i, \gamma}^{\alpha}\right)_{i=1}^{2 m} .
$$

Since $\operatorname{dim}\left(\mathcal{H}_{m, \gamma}^{\alpha}\right)<\infty$, there exists a best approximation $f_{m, \gamma}^{\alpha}$ of $f \in \mathcal{C}[-\pi, \pi]$ in $\mathcal{H}_{m, \gamma}^{\alpha}$. The subspace $\mathcal{H}_{m, \gamma}^{\alpha}$ is closed.
Let us define

$$
d_{m, \gamma}^{\alpha}(f)=\left\|f-f_{m, \gamma}^{\alpha}\right\|_{\infty} .
$$

$\mathcal{H}_{m, \gamma}^{\alpha}$ is fundamental for $\gamma>2$ fixed, since for any $\epsilon>0$ and $f \in \mathcal{C}[-\pi, \pi]$, according to Theorem 3.2, there exists a linear combination of elements of the set $\left\{h_{m, i, \gamma}^{\alpha}: i=1,2, \ldots, 2 m\right\}$ such that the distance to $f$ is lower than $\epsilon$. Thus, $\mathcal{H}_{m, \gamma}^{\alpha}$ is complete, since in a Banach space every fundamental set is complete. 12

Let us define

$$
\mathcal{H}_{\gamma}^{\alpha}=\operatorname{span}\left(\mathcal{H}_{m, \gamma}^{\alpha}\right)_{m=1}^{\infty} .
$$

In the case $\gamma=4$ and $\alpha=0, \mathcal{H}_{4}$ is a set of trigonometric polynomials, $\frac{19}{19}$ henceforth $\mathcal{H}_{\gamma}^{\alpha}$ is a double generalization of this kind of mappings (with respect to the exponent $\gamma$ and the scale $\alpha$ ). 
The operator $D_{m, \gamma}^{\alpha}: \mathcal{C}[-\pi, \pi] \rightarrow \mathcal{C}[-\pi, \pi]$ is linear (3.1) and

$$
\begin{aligned}
\left\|D_{m, \gamma}^{\alpha}(f)\right\|_{\infty} & \leq \sum_{i=1}^{2 m}\left|f\left(x_{i}\right) \| h_{m, i, \gamma}^{\alpha}(x)\right| \\
& \leq\|f\|_{\infty}\left\|H_{m, \gamma}\right\|_{\infty} \sum_{i=1}^{2 m}\left|P_{m, i, \gamma}^{\alpha}(x)\right| .
\end{aligned}
$$

From (3.16), the norm of the operator $D_{m, \gamma}^{\alpha}$ with respect to the sup-norm in $\mathcal{C}[-\pi, \pi]$ can be bounded as

$$
\left\|D_{m, \gamma}^{\alpha}\right\| \leq \frac{1}{2}\left(\frac{\pi}{2}\right)^{\gamma} \Lambda_{m, \gamma}^{\alpha}
$$

where $\Lambda_{m, \gamma}^{\alpha}$ is the Lebesgue constant associated to the nodal kernels $\left\{P_{m, i, \gamma}^{\alpha}\right\}_{i=1}^{2 m}$.

For $\alpha=0$, i.e. in the classical case, since $\sum_{i=1}^{2 m} h_{m, i, \gamma}(x)=H_{m, \gamma} H_{m, \gamma}^{-1}=1$ according to (3.2),

$$
\left\|D_{m, \gamma}(f)\right\|_{\infty} \leq\|f\|_{\infty}
$$

and consequently

$$
\left\|D_{m, \gamma}\right\| \leq 1
$$

The condition of the function values is measured by $\left\|D_{m, \gamma}\right\|$, because

$$
\begin{aligned}
\left\|D_{m, \gamma}(f)-D_{m, \gamma}(\widetilde{f})\right\|_{\infty} & \leq\left\|D_{m, \gamma}\right\|, \\
\|f-\widetilde{f}\|_{\infty} & \leq\|f-\widetilde{f}\|_{\infty} .
\end{aligned}
$$

The operator increases the errors for no value of $\gamma$ and $m$ (see Table 1 ).

Lemma 4.1. If $\gamma>2,1 \in \sigma_{p}\left(D_{m, \gamma}\right)$, where $\sigma_{p}\left(D_{m, \gamma}\right)$ is the point spectrum of $D_{m, \gamma}$.

Proof. Let us consider $f(x)=1$ for any $x \in[-\pi, \pi]$. The definition of $D_{m, \gamma}$ implies that $D_{m, \gamma}(f)=1=f$, and $1 \in \sigma_{p}\left(D_{m, \gamma}\right)$.

Proposition 4.1. For $\gamma>2$ and any $m \in N$, $\left\|D_{m, \gamma}\right\|=1$.

Proof. The inequality $\left\|D_{m, \gamma}\right\| \leq 1$ is already proved (4.1). For the other, if we take $f=1$,

$$
\frac{\left\|D_{m, \gamma}(f)\right\|_{\infty}}{\|f\|_{\infty}}=1
$$

according to the previous lemma, and thus $1 \leq$ $\left\|D_{m, \gamma}\right\|$.
The operator $D_{m, \gamma}$ does not amplify errors in $\mathcal{L}^{2}$ sense either since

$$
\begin{aligned}
\left\|D_{m, \gamma}(f)\right\|_{2}^{2} & =\frac{1}{2 \pi} \int_{-\pi}^{\pi}\left|D_{m, \gamma}(f)\right|^{2} d x \\
& \leq\left\|D_{m, \gamma}(f)\right\|_{\infty}^{2} \leq\|f\|_{\infty}^{2} .
\end{aligned}
$$

Consequently

$$
\left\|D_{m, \gamma}(f)-D_{m, \gamma}(\widetilde{f})\right\|_{2} \leq\|f-\widetilde{f}\|_{\infty},
$$

where $\tilde{f}$ is the perturbed function of $f$.

\section{ACKNOWLEDGMENTS}

Sangita Jha acknowledges the partial financial support received from IIT Madras to visit the University of Zaragoza, Spain.

\section{REFERENCES}

1. P. E. T. Jorgensen, Analysis and Probability: Wavelets, Signals, Fractals, Graduate Texts in Mathematics, Vol. 234 (Springer, New York, 2006).

2. M. F. Barnsley and A. N. Harrington, The calculus of fractal interpolation functions, J. Approx. Theory $\mathbf{5 7}(1)$ (1989) 14-34.

3. A. K. B. Chand and G. P. Kapoor, Generalized cubic spline fractal interpolation functions, SIAM J. Numer. Anal. 44(2) (2006) 655-676.

4. M. A. Navascués and M. V. Sebastián, Smooth fractal interpolation, J. Inequal. Appl. 2006 (2006) 78734 .

5. M. F. Barnsley, Fractal functions and interpolation, Constr. Approx. 2(4) (1986) 303-329.

6. M. F. Barnsley, Fractals Everywhere (Academic Press, Boston, 1988).

7. M. A. Navascués and A. K. B. Chand, Fundamental sets of fractal functions, Acta Appl. Math. 100(3) (2008) 247-261.

8. P. Viswanathan, A. K. B. Chand and M. A. Navascués, Fractal perturbation preserving fundamental shapes: Bounds on the scale factors, J. Math. Anal. Appl. 419(2) (2014) 804-817.

9. P. Viswanathan, M. A. Navascués and A. K. B. Chand, Associate fractal functions in $\mathcal{L}^{p}$-spaces and in one-sided uniform approximation, J. Math. Anal. Appl. 433(2) (2016) 862-876.

10. P. Viswanathan, M. A. Navascués and A. K. B. Chand, Fractal polynomials and maps in approximation of continuous functions, Numer. Funct. Anal. Optim. 37(1) (2016) 106-127.

11. D. Jackson, On approximation by trigonometric sums and polynomials, Trans. Amer. Math. Soc. 13(4) (1912) 491-515.

12. P. J. Davis, Interpolation and Approximation (Dover, New York, 1975). Republication, with minor 
corrections, of the 1963 original, with a new preface and bibliography.

13. M. A. Navascués, Fractal polynomial interpolation, Z. Anal. Anwend. 24(2) (2005) 401-418.

14. M. A. Navascues, Fractal trigonometric approximation, Electron. Trans. Numer. Anal. 20 (2005) 6474.

15. E. W. Cheney, Introduction to Approximation Theory (AMS Chelsea Publishing, Providence, 1998). Reprint of the second (1982) edition.

16. C. W. Ueberhuber, Numerical computation 2: Methods, Software, and Analysis (Springer-Verlag, Berlin, 1997). Translated and revised from the 1995 German original.

17. G. Hämmerlin and K. H. Hoffmann, Numerical Mathematics, Readings in Mathematics, (SpringerVerlag, New York, 1991). Translated from the
Original German edition published as Band 7 of the series: Grundwissen Mathematik by L. L. Schumaker.

18. T. J. Rivlin, Chebyshev Polynomials: From Approximation Theory to Algebra and Number Theory, 2nd edn., Pure and Applied Mathematics (John Wiley \& Sons, New York, 1990).

19. D. Jackson, On the accuracy of trigonometric interpolation, Trans. Amer. Math. Soc. 14(4) (1913) 453-461.

20. N. I. Achieser, Theory of Approximation (Dover, New York, 1992). Translated from the Russian and with a preface by Charles J. Hyman; Reprint of the 1956 English translation.

21. G. G. Lorentz, Approximation of Functions, 2nd edn. (Chelsea, New York, 1986). 